\title{
Aphid sex pheromone components: age-dependent release by females and species-specific male response
}

\author{
Jim Hardie ${ }^{1 *}$, Marcel Holyoak ${ }^{1}$, Jill Nicholas ${ }^{1}$, Stephen F. Nottingham ${ }^{1}$, John A. Pickett ${ }^{2}$, \\ Lester J. Wadhams ${ }^{2}$, and Christine M. Woodcock ${ }^{2}$ \\ 1 Agricultural and Food Research Council Link Research Group in Aphid Biology, Department of Biology, Imperial \\ College of Science, Technology and Medicine, London SW7 2AZ, United Kingdom \\ 2 AFRC Institute of Arable Crops Research, Rothamsted Experimental Station, Harpenden, Herts AL5 2JQ, United \\ Kingdom
}

Received June 6, 1990 / Accepted July 10, 1990

\section{Summary}

Sex pheromone released by Megoura viciae oviparae was collected and analysed for the quantity and relative amounts of each of the two biologically active components. Pheromone was not produced until the second day of the adult stadium, increasing in quantity up to day 6 and then declining. The ratio of $(-)-(1 R, 4 \mathrm{a} S, 7 S, 7 \mathrm{a} R)$-nepetalactol: $(+)-(4 a S, 7 S, 7 a R)$-nepetalactone pheromone mixture changed from $c a 1: 5$ on days 2-6 after adult moult to $1: 12$ on days 7 and 8 , but returned to the original composition by day 10 .

The behaviour of male aphids from three species, Acyrthosiphon pisum, Aphis fabae, and $M$. viciae, was observed in an olfactometer and in two mating bioassays, using both synthetic and natural sex pheromone. The two active components were combined in ratios which mimicked the pheromone released by these and other aphid species. It was found that:

1. $M$. viciae males were attracted by the synthetic sex pheromone in the olfactometer, with maximal attraction to the conspecific ratio. On the other hand, $A$. pisum and $A$. fabae males did not respond to any pheromone mixtures in the olfactometer.

2. Males of all three species could be induced to attempt co- pulation with conspecific virginoparae treated with synthetic pheromone (virginoparae reproduce asexually and do not produce the sex pheromone). The response in $M$. viciae and $A$. fabae was equivalent to that elicited by natural pheromone released by calling conspecific oviparae, but in $A$. pisum it was slightly lower.

3. In $M$. viciae, the relative activities of the nepetalactol and the nepetalactone were reversed in the two assays. Thus the former was more potent in the mating bioassay, the latter in the olfactometer. The nepetalactol appeared to act as an aphrodisiac and the nepetalactone as an attractant.

4. Mating response was greatest at the species-specific ratio of the two components, but males were also significantly responsive to mixtures mimicking the sex pheromone of other species.

The results are discussed in relation to mate seeking, together with the mechanisms involved in speciesspecific mating.

\section{Key words}

conspecific mating, attraction, sex pheromone, olfactometer, nepetalactol, nepetalactone, Homoptera, Aphidae, Acyrthosiphon pisum, Aphis fabae, Megoura viciae

\section{Introduction}

Pettersson (1970a, 1971) and Marsh (1972) first observed that sexual female aphids (oviparae) released a sex pheromone which attracted males. The pheromone was released from scent plaques on the hind tibiae and detected by olfactory sensilla on male antennae. They also reported that

\footnotetext{
* Address for correspondence: Dr Jim Hardie, AFRC Link Group in Aphid Biology, Imperial College at Silwood Park, Ascot, Berks SL5 7PY, United Kingdom
}

(c) Georg Thieme Verlag Stuttgart - New York attraction was restricted to a short distance $(<10 \mathrm{~cm})$, release was confined to the light phase of the light/dark cycle and that the underlying rhythm of release was endogenous. It was also noted that males of one species would sometimes attempt to mate with females of a different species, although recent studies have indicated that males can differentiate between biotypes of the same species (Eisenbach \& Mittler 1987).

The chemical identity of the sex pheromone of the vetch aphid, Megoura viciae, was determined by Dawson et al. (1987, 1989). Two components, specific isomers of the monoterpenoids nepetalactol and nepetalactone, were physiologically active on olfactory neurones associated with the secondary rhinaria of the male. In olfactometer-based bioassays, males responded to a mixture of the compounds 
but not to either compound presented alone. It has now been shown that oviparae from a number of different aphid species release the same two compounds, but that the relative quantities differ. Thus, the greenbug, Schizaphis graminum, released an 8:1 ratio of nepetalactol: nepetalactone (Dawson et al. 1988) and in other species, this ratio varied between 2:1 and 1:29 (see below and Dawson et al. 1990).

In this paper, the quantity and ratio of these two components released by female $M$. viciae are measured. Furthermore, the response of males of three aphid species to calling, conspecific oviparae (i.e. females releasing pheromone) and to synthetic mixtures designed to mimic the sex pheromone released by various species is reported. Olfactometer studies reveal chemotactic responses, while pheromonetreated "model" oviparae demonstrate the interaction of visual and chemical stimuli leading to mating.

\section{Materials and methods}

\section{Insects}

Three aphid species were chosen for investigation, the pea aphid Acyrthosiphon pisum (Harris), the black bean aphid Aphis fabae (Scop.), and the vetch aphid Megoura viciae (Buckt.). All were reared on broad bean, Vicia faba (Moench) at $15^{\circ} \mathrm{C}$ and the winged males and apterous oviparae were induced by short photoperiods which varied with species. Thus, in the pea aphid, male production was favoured at LD 13:11 and ovipara production at LD 12:12, while LD 12:12 induced both sexes of the black bean and vetch aphids. Where necessary, the sexes were separated as late-stadia larvae so that all experimental insects were virgin.

\section{Release of pheromone by oviparae of M. viciae}

The sex pheromone released by $M$. viciae oviparae was collected over the first ten days after adult moult. Volatiles from twenty oviparae feeding on beans were collected on Porapak Q (Blight 1990). This was replaced every 24 $\mathrm{h}$, using the same oviparae, and the volatiles then eluted with ether. The samples were concentrated to $100 \mu \mathrm{l}$ under a stream of nitrogen at room temperature and undecane was added as an internal standard. The extracts were analysed by gas chromatography on a $50 \mathrm{~m} \times 0.32 \mathrm{~mm}$ i.d. HP-1 fused silica capillary column equipped with a cold on-column injector and with hydrogen as the carrier gas.

\section{Chemotaxis}

Studies were carried out at room temperature $\left(20-25^{\circ} \mathrm{C}\right)$ in a linear track olfactometer. The apparatus was similar to that used to detect chemotaxis in cockroaches (Sakuma \& Fukami 1985), but modified for aphids. A group of 15-25 aphids was placed in a teflon-coated dish in which the vertical bar of a T-wire walk-way was positioned. Air was drawn through the apparatus at a rate of 1 litre per minute. The surrounding arrangement of plexiglass tubing ensured that air was drawn in through two side chambers into which test odours could be introduced. The air-flows met at the intersection of the vertical and horizontal wires of the T. Thus, as the insects, attracted by an overhead light $(60 \mathrm{~W}$ tungsten bulb), reached the top of the vertical wire, they could choose to move horizontally in only one of two directions. Both di- rections were upwind but the olfactory stimuli encountered in the air-flows could be manipulated experimentally. Aphid behaviour was observed over a ten minute period and the numbers of insects moving, at least one body-length, towards either side chamber were noted. Observations where no insects climbed the vertical wire during the first ten minute period were ignored.

$(-)-(1 R, 4 \mathrm{aS}, 7 S, 7 \mathrm{aR})-$ Nepetalactol, $\quad(+)-(4 \mathrm{a} S$, $7 S, 7 \mathrm{a} R$ )-nepetalactone (Dawson et al. 1989) and mixtures of the two, dissolved in $10 \mu 1$ hexane, were applied to glass cover slips and the hexane evaporated prior to testing against a similarly treated solvent control. The synthetic pheromone mixtures mimicked sex pheromone released by a number of aphid species. The ratios of nepetalactol:nepetalactone previously recorded were 8:1 (S. graminum), 2:1 (Myzus persicae), 1:1 (A. pisum), 1:4 (young $M$. viciae), 1:12 (day $8 M$. viciae), and 1:29 (A. fabae) (Dawson et al. 1988, 1990, this paper). There was some variation in the ratio recorded in young $M$. viciae, namely between 1:4 and 1:6; for the present experiments, 1:4 was chosen.

\section{Mating}

After allowing individual oviparae to settle on their host plants and begin calling, males were walked closely by them. Oviparae of $A$. pisum and $M$. viciae readily settled on young bean seedlings and for $A$. fabae studies, detached leaves of the winter host, spindle (Euonymus europaeus L.), were used. For each male, five trials were carried out in succession, each trial ending when copulation was attempted or when the male had settled in one position for two minutes. Attempted copulation was defined as the male climbing onto the female and correctly aligning its body prior to flexing the abdomen ventrally to copulate. At this stage, the male was gently removed and the next trial started. If contact was made, males were given two minutes in which to attain the mating position prior to removal. Males were between 1 and 21 days old, females less than $24 \mathrm{~h}$ or ca 6 days old and all trials were carried out between photophase hours 2 and 7 . These ages and times were chosen to optimize the experimental conditions (Marsh 1972, 1975, Eisenbach \& Mittler 1980). Male $A$. fabae proved flighty and their wings were clipped prior to assay.

The response of males to pheromone components was tested in a similar way, using parthenogenetic females (virginoparae) as "model" oviparae. These morphs do not release the sex pheromone (Marsh 1975), but are visually similar to oviparae. Adult conspecific virginoparae provided "model" oviparae for $A$. pisum and $M$. viciae, while third stadia $M$. viciae larvae and adult $A$, fabae virginoparae were used to model oviparae of $A$. fabae. Acetone solutions $(0.1$ $\mu \mathrm{l})$ of nepetalactol and nepetalactone at 20 and $2 \mathrm{ng}$, and mixtures of the two compounds, were topically applied to the dorsal abdominal surface and the treated virginoparae were tested for their attractiveness to male aphids. Preliminary experiments indicated that the pheromone components were effective over a period of thirty minutes and trials were completed within this time. In each of the above mating assays there were a hundred trials with twenty males. 
Table 1 Numbers of male Megoura viciae that responded to eight different mixtures of the two sex pheromone components in the olfactometer

\begin{tabular}{|c|c|c|c|c|c|}
\hline $\begin{array}{l}\text { Ratio } \\
\text { ol:one }\end{array}$ & Species mimicked & $\begin{array}{l}\text { Replicates } \\
\text { (n) }\end{array}$ & $\begin{array}{l}\text { Results } \\
\text { Treatment } \\
\bar{X} \pm S E M\end{array}$ & $\begin{array}{l}\text { Control } \\
X \pm S E M\end{array}$ & \\
\hline $\begin{array}{l}1: 0 \\
0: 1 \\
8: 1 \\
2: 1 \\
1: 1 \\
1: 4 \\
1: 12 \\
1: 29\end{array}$ & $\begin{array}{l}- \\
- \\
\text { S. graminum } \\
\text { M. persicae } \\
\text { A. pisum } \\
\text { M. viciae }\{\text { day } 2-6\} \\
\text { M. viciae (day } 7-8\} \\
\text { A. fabae }\end{array}$ & $\begin{array}{l}6 \\
7 \\
8 \\
7 \\
7 \\
8 \\
6 \\
6\end{array}$ & $\begin{array}{l}2.3 \pm 1.3 \\
2.6 \pm 0.4 \\
2.3 \pm 0.6 \\
2.4 \pm 0.7 \\
2.9 \pm 0.9 \\
3.9 \pm 0.6 \\
2.2 \pm 0.4 \\
2.2 \pm 0.6\end{array}$ & $\begin{array}{l}1.2 \pm 0.7 \\
0.1 \pm 0.1 \\
0.8 \pm 0.3 \\
0.9 \pm 0.5 \\
1.4 \pm 0.9 \\
0.3 \pm 0.2 \\
0.7 \pm 0.3 \\
1.2 \pm 0.5\end{array}$ & $\begin{array}{l}\text { ns } \\
{ }^{*} \\
\text { ns } \\
\text { ns } \\
\text { ns } \\
* \\
* \\
\text { ns }\end{array}$ \\
\hline $0: 0$ & Hexane control & 8 & $0.9 \pm 0.3$ & $1.7 \pm 0.5$ & ns \\
\hline
\end{tabular}

Ratio of nepetalactol: nepetalactone; 2 ng applied

* $P<0.05$ Mann-Whitney U test

ns Not significant

Table 2 Attempted matings of males with female morphs

\begin{tabular}{|c|c|c|c|c|}
\hline $\begin{array}{l}\text { Female } \\
\text { Species }\end{array}$ & morph & age/condition & $\begin{array}{l}\text { Male } \\
\text { Species }\end{array}$ & $\begin{array}{l}\text { Percentage attempted } \\
\text { copulation }\end{array}$ \\
\hline $\begin{array}{l}\text { M. viciae } \\
\text { M. viciae } \\
\text { M. viciae }\end{array}$ & $\begin{array}{l}\text { ovipara } \\
\text { ovipara } \\
\text { ovipara }\end{array}$ & $\begin{array}{l}\text { adult-calling } \\
\text { adult-calling } \\
\text { adult-calling }\end{array}$ & $\begin{array}{l}\text { M. viciae } \\
\text { A. pisum } \\
\text { A. fabae }\end{array}$ & $\begin{array}{r}94 \\
22 \\
0\end{array}$ \\
\hline $\begin{array}{l}\text { M. viciae } \\
M . \text { viciae } \\
M . \text { viciae }\end{array}$ & $\begin{array}{l}\text { ovipara } \\
\text { ovipara } \\
\text { ovipara }\end{array}$ & $\begin{array}{l}\text { adult- }<24 \mathrm{~h} \\
\text { adult- }<24 \mathrm{~h} \\
\text { adult- }<24 \mathrm{~h}\end{array}$ & $\begin{array}{l}\text { M. viciae } \\
\text { A. pisum } \\
\text { A. fabae }\end{array}$ & $\begin{array}{l}7 \\
0 \\
0\end{array}$ \\
\hline $\begin{array}{l}\text { A. pisum } \\
\text { A. fabae }\end{array}$ & $\begin{array}{l}\text { ovipara } \\
\text { ovipara }\end{array}$ & $\begin{array}{l}\text { adult-calling } \\
\text { adult-calling }\end{array}$ & $\begin{array}{l}\text { A. pisum } \\
\text { A. fabae }\end{array}$ & $\begin{array}{l}69 \\
42\end{array}$ \\
\hline $\begin{array}{l}\text { M. viciae } \\
\text { M. viciae } \\
\text { M. viciae }\end{array}$ & $\begin{array}{l}\text { virginopara } \\
\text { virginopara } \\
\text { virginopara }\end{array}$ & $\begin{array}{l}\text { adult } \\
\text { adult } \\
\text { adult }\end{array}$ & $\begin{array}{l}\text { M. viciae } \\
\text { A. pisum } \\
\text { A. fabae }\end{array}$ & $\begin{array}{r}10 \\
9 \\
0\end{array}$ \\
\hline M. viciae & virginopara & larva-3rd stadium & A.fabae & 0 \\
\hline A. fabae & virginopara & adult & A. fabae & 0 \\
\hline
\end{tabular}

\section{Results}

\section{Pheromone release by oviparae of $M$. viciae}

The volatiles collected over 24-h periods during the ten days after adult moult were analysed for levels of the nepetalactol and the nepetalactone (Fig. 1). On day 1, the combined amount of both compounds was negligible but increased steadily up to day 6 , after which it declined. During this period, the ratio of the two components was not constant but changed from $c a 1: 5$ on days $2-6$ to $c a 1: 12$ on days 7-8, before returning to the original $c a 1: 5$ ratio by day 10 .

\section{Chemotaxis}

The responses of male $M$. viciae in the olfactometer to $2 \mathrm{ng}$ of the pheromone components at different ratios are shown in Table 1. There was an attraction to ratios close to those released by conspecific oviparae and to nepetalactone alone, but nepetalactol neither attracted nor repelled. In the hexane controls, equal numbers of insects moved towards both side chambers. At higher concentrations (200 and
$20 \mathrm{ng}$ ), males were attracted to each mixture as well as to nepetalactone, but not to nepetalactol.

Male A. fabae and $A$. pisum did not respond in the olfactometer to the pheromone components alone, or to mixtures.

\section{Mating}

The proportion of males attempting to copulate with calling conspecific oviparae varied with species. $M$. viciae was again the most responsive species, with $94 \%$ of trials resulting in attempted mating ( $\mathrm{n}=20$ males; 100 trials). A. fabae scored in only $42 \%$ of trials, while $A$. pisum had an intermediate score of $69 \%$ (Table 2). Adult virginoparae of $M$. viciae could induce a small proportion of conspecific and $A$. pisum males to attempt copulation, presumably via visual cues alone. Male $A$. fabae, however, did not attempt copulation with larval or adult $M$. viciae virginoparae nor with conspecific adult virginoparae. 


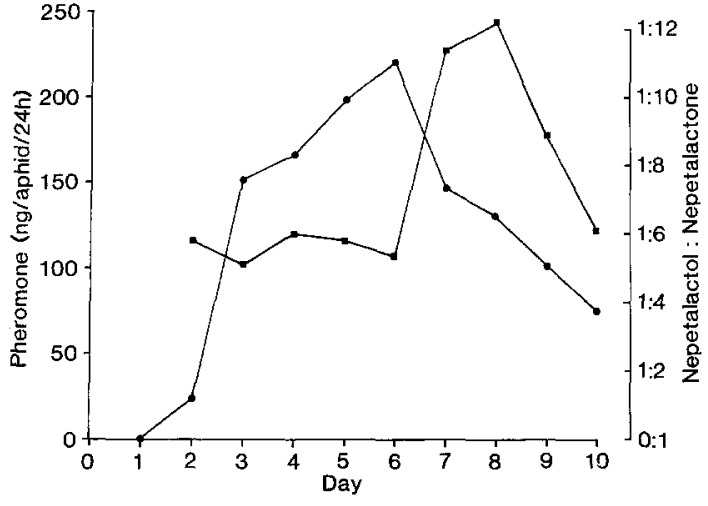

Fig. 1 Pheromone production by oviparae of Megoura viciae over a ten day period following the final moult. $\bullet$-amount of pheromone produced; m-ratio of nepetalactol: nepetalactone during emission

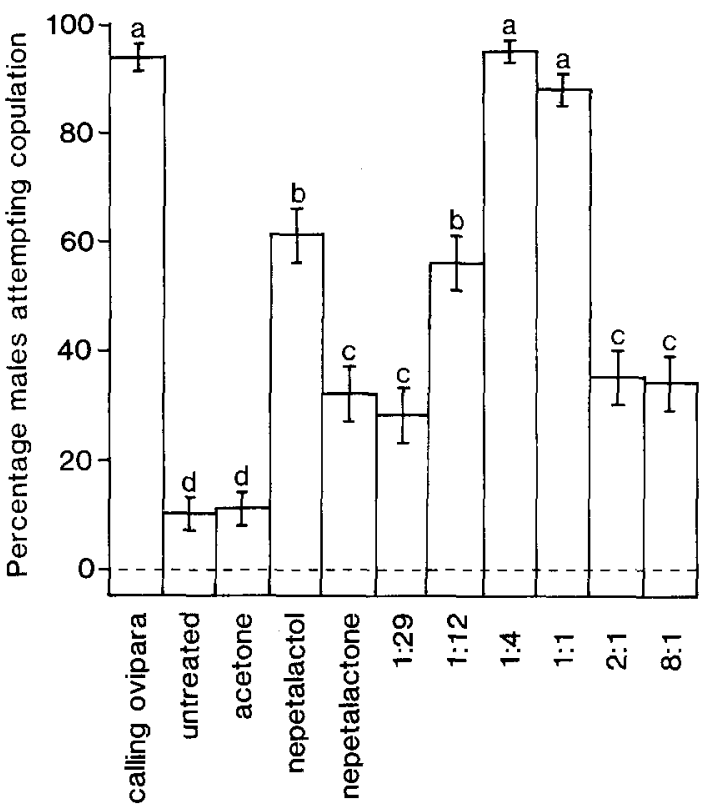

Fig. 2 Mating bioassay - Megoura viciae. Treatments refer to a calling conspecific ovipara or 2 ng of synthetic pheromone applied topically to the abdomen of adult conspecific virginoparae; ratios are amounts of nepetalactol:nepetalactone. Controls were untreated or treated with $0.1 \mu \mathrm{l}$ acetone. Letters represent significant differences, $P<0.05$, Chisquared test; bars $= \pm \$ E M$

Virginoparae treated with various mixtures and concentrations of pheromone components were used as "model" oviparae and the mating behaviour of the males was compared with that elicited by calling conspecific oviparae (Figs. 2, 3, 4). For each species, mixtures of nepetalactol and nepetalactone induced significantly more mating attempts than the acetone solvent alone, but maximum attraction was achieved by the ratio of the components equivalent to that released by calling conspecific oviparae. Indeed, for $M$. viciae, 2 ng of synthetic pheromone in the conspecific ratio (1:4) was as effective at enticing the males as a calling female. For $A$. pisum, 2 ng of the conspecific ratio $(1: 1)$ was almost as attractive as a calling female, while for $A$. fabae, $20 \mathrm{ng}$ of the conspecific ratio $(1: 29)$ was required. When tested individually, the pheromone components did not significantly increase mating attempts in $A$. pisum or $A$. fabae but were effective in $M$. viciae.

The proportion of attempted copulations by male $A$. pisum with untreated $M$. viciae virginoparae could be increased from $9 \%$ (Table 2) to $23 \%$ and $22 \%$ with applications of 2 or $20 \mathrm{ng}$ (respectively) of a 1:1 ratio of nepetalactol:nepetalactone. However, $20 \mathrm{ng}$ of pheromone mimicking $A$. fabae, applied to adult or third stadia virginoparae of $M$. viciae, did not increase their attractiveness to male $A$. fabae (cf. Table 2).

\section{Discussion}

In the olfactometer bioassays of Marsh (1972, 1973) using $M$. viciae, attractancy of oviparae to males increased from day 2 to day 6 and declined from then on to day 14. There was both a change in the proportion of males responding during the photophase and in the numbers of hours over which male activity was initiated. This was thought to reflect changes in the amount of sex pheromone released by the oviparae. In the current study, when pheromone was collected over $24-h$ periods, it was shown that not only did pheromone quantity depend on the age of the ovipara, but so did the ratio of the components. Maximal release occurred on day 6 , as did the maximal response of Marsh's walking males. The mating bioassay revealed a significant difference between the response to $1: 4$ and $1: 12$ ratios (Fig. 2). However, both ratios were attractive in the olfactometer (Table 1). Thus, it appears that Marsh's quantification of pheromone production using the response of males in an olfactometer holds true, but that mating attempts would be reduced on days 7 and 8 when the ratio of the two components changes ( $c f$. Fig. 2). The discrepancy between a previous olfactometer assay where neither pheromone component was attractive on its own (Dawson et al. 1987) and the present demonstration of attraction to nepetalactone may be due to different olfactometer design. It is also noteworthy that close inspection of the previous results shows figures not far from significant for a nepetalactone attraction (Dawson et al. 1987).

Electrophysiological studies have shown that the pheromone is perceived by separate olfactory cells on the male antenna which respond preferentially to either nepetalactol or nepetalactone (Dawson et al. 1990). This ability to discriminate between the pheromone components is reflected in the behavioural preference of males for the conspecific ratio. $M$. viciae males in the olfactometer showed an attraction to their species-specific ratios and to nepetalactone alone (Table 1) suggesting that nepetalactone may act as an attractant. Nepetalactol appeared to inhibit this attraction (even at ratios as low as 1:29), expect when the ratio of the two components was close to that released by the conspecific female and the attraction was significantly enhanced. However, in the mating assays, nepetalactol was significantly more active than nepetalactone, suggesting that it is acting as an aphrodisiac to induce copulation. In $M$. viciae and $A$. fabae, the overall mating response of the species to their own calling females was mimicked by applying synthetic mixtures of nepetalactol and nepetalactone to conspecific virginoparae. However, in A. pisum, the response to pheromone-treated virginoparae was significantly less than to calling oviparae and other factors may be involved. Visual stimuli, for example, are known to affect 
mating behaviour (Pettersson 1971, Marsh 1975). In the current experiments, green simulated "oviparae" in the form of pheromone-treated virginoparae of $M$. viciae (third stadia larvae and adults) were not attractive to $A$. fabae males but black simulated "oviparae", in the form of pheromonetreated adult $A$. fabae virginoparae, were attractive. Similarly, $M$. viciae virginoparae may have been poor ovipara-mimics for $A$. pisum males since although they were of similar size, they were darker green in colour.

It has been noted previously that the olfactory attraction of male aphids to calling oviparae was not necessarily species-specific (Pettersson 1971, Marsh 1975). The recent finding that the sex pheromone of a number of aphid species, from two tribes, contain the same two active components provides an explanation for these observations (Dawson et al. $1987,1988,1990)$. Indeed, the ratios of the nepetalactol and the nepetalactone in $A$. pisum and $M$. viciae are similar (i.e. $1: 1$ and $c a 1: 4$, respectively), although the present results indicate that $M$. viciae males may be more responsive to the $A$. pisum ratio than vice versa (Figs 2 and 3 ). The current work also demonstrates that calling oviparae of $M$. viciae can provoke limited mating attempts by male $A$. pisum.

In Lepidoptera, species specificity of pheromones is more precise and achieved by different individual chemicals and by specific blends of chemicals (Tamaki 1985). Species-specific mating in aphids may rely more on spatial and temporal separation than on pheromonal specificity. The role of the host plant may well be crucial, particularly in hostalternating species which tend to be monophagous with regard to the primary host. The sexual females are wingless, their location depending on the larviposition preference of their mothers, and the evolution of a well developed species specificity in pheromones may therefore be less necessary. The pheromonal similarity between the monoecious (non host-alternating) A. pisum and $M$. viciae is perhaps surprising, as they can occur on the same host plant (Blackman \& Eastop 1985). In this case, there may well be a temporal separation between the sexual forms. It has been found that the critical night length for the induction of oviparae in two English clones differs by $1.5 \mathrm{~h}(11$ vs $9.5 \mathrm{~h}$, Lees 1973,1989$)$. In the field, $M$. viciae would produce oviparae some three weeks earlier than A. pisum. However, although the two species may attempt copulation, this does not result in sperm transfer, presumably because of incompatibility of the genitalia (Hardie and Holyoak unpublished). Species-specific mating will also depend upon visual cues such as colour, shape, size (see above) and possibly on specific movements by the ovipara during calling, as suggested by Steffan (1990).

The precise role of the sex pheromone in aphids is still unclear. In host-alternating species in particular, the mate-seeking male would be greatly facilitated by a longdistance, pheromone-mediated orientation as observed in male Lepidoptera (Cardé 1984). An alternative strategy is for the male firstly to locate the primary host plant and then to search locally for receptive females. The precise sensory cues responsible for host-plant selection are not known (Klingauf 1987), but there is evidence that heteroecious males are attracted by primary host odour (Tamaki et al. 1970; Pettersson 1970b). There were thought to be constraints, however, as aphid males (like females) are weak fliers and could not make headway in wind speeds above $c a 0.8 \mathrm{~m} / \mathrm{s}$ (Kennedy \& Tho-

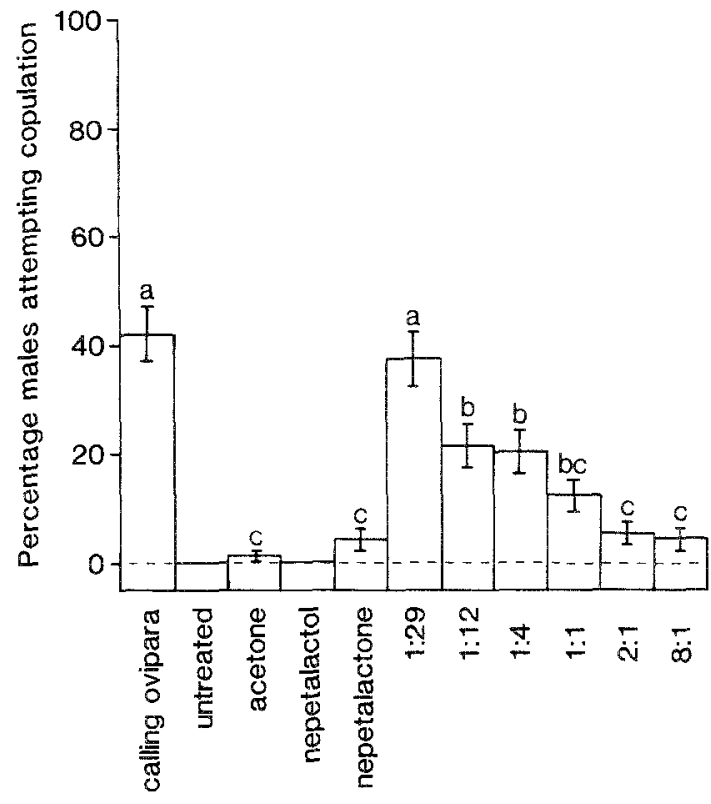

Fig. 3 Mating bioassay - Acyrthosiphon pisum. Details as in Figure 2 . with adult virginoparae of $A$. pisum being used as "model" oviparae

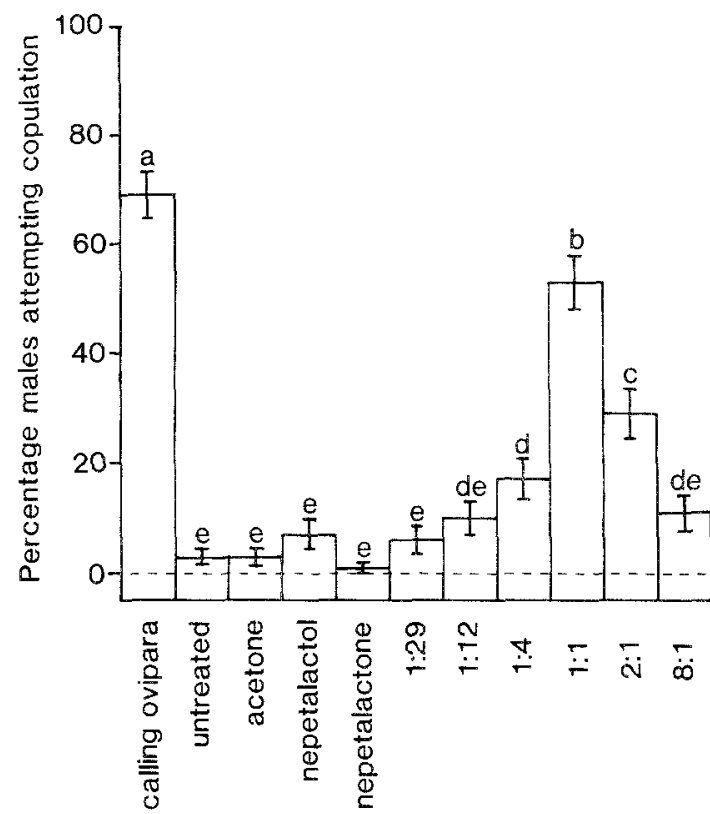

Fig. 4 Mating bioassay - Aphis fabae. Details as in Figure 2, but with $20 \mathrm{ng}$ of synthetic pheromone apolied to adult conspecific virginoparae of $A$. fabae acting as "model" oviparae

mas 1974) and even with clusters of calling oviparae, the supposed short-range action of the sex pheromone (Pettersson 1971; Marsh 1975) indicated that the chances of a successful search of a large primary host would seem remote. Recent field experiments with the damson-hop aphid, Phorodon humuli, have shown that these laboratory-determined constraints may have been overestimated. $P$. humuli males were selectively caught in water traps incorporating a synthetic sex pheromone lure, demonstrating that these small insects can indeed locate an odour source under field conditions (Campbell 
et al. 1990). In addition, the male catches were significantly enhanced in traps containing both sex pheromone and hostplant volatiles, again emphasising the co-rôles of pheromone and host plant in effecting species-specific recognition.

\section{References}

Blackman RL, Eastop VF (1985) Aphids on the World's Crops: An Identification Guide. Chichester: John Wiley and Sons

Blight MM (1990) Techniques for isolation and characterisation of volatile semiochemicals of phytophagous insects. Proceedings of the First International Symposium on the Chromatography and Isolation of Insect Hormones, Pheromones and Related Compounds, Reading 1989. In press

Campbell CAM, Dawson GW, Griffiths DC, Pettersson J, Pickett JA, Wadhams LJ, Woodcock CM (1990) The sex attractant pheromone of the damson-hop aphid Phorodon humuli (Homoptera, Aphididae) J Chem Ecol in press

Cardé RT (1984) Chemo-orientation in flying insects. Pp 111-124 in Bell WJ \& Cardé RT (eds) Chemical Ecology of Insects. London: Chapman and Hall

Dawson GW, Griffiths DC, Janes NF, Mudd A, Pickett JA, Wadhams LJ, Woodcock CM (1987) Identification of an aphid sex pheromone. Nature 325:614-616

Dawson GW, Griffiths DC, Merritt LA, Mudd A, Pickett JA, Wadhams LJ, Woodcock CM (1988) The sex pheromone of the greenbug, Schizaphis graminum. Entomol exp appl 48:91-93

Dawson GW, Janes NF, Mudd A, Pickett JA, Slawin AMZ, Wadhams LJ, Williams DJ (1989) The aphid sex pheromone. Pure appl Chem 61:555-558

Dawson GW, Griffiths DC, Merritt LA, Mudd A, Pickett JA, Wadhams LJ, Woodcock CM (1990) Aphid semiochemicals - a review, and recent advances on the sex pheromone. $\mathbf{J}$ Chem Ecol, in press

Eisenbach J, Mittler TE (1980) An aphid circadian rhythm: factors affecting the release of sex pheromone by oviparae of the greenbug, Schizaphis graminum. J Insect Physiol 26:511-516
Eisenbach J, Mittler TE (1987) Sex pheromone discrimination by male aphids of a biotype of Schizaphis graminum. Entomol exp appl 43:181182

Kennedy JS, Thomas AAG (1974) Behaviour of some low-flying aphids in wind. Ann appl Biol 76:143-159

Klinghauf FA (1987) Host plant finding and acceptance. Pp 209-233 in Minks AK \& Harrewijn P (eds) Aphids, their Biology, Natural Enemies and Control. Vol. 2A. Amsterdam: Elsevier

Less AD (1973) Photoperiodic time measurement in the aphid Megoura viciae. J Insect Physiol 19:2279-2316

Lees AD (1989) The photoperiodic responses and phenology of an English strain of the pea aphid Acyrthosiphon pisum. Ecol Entomol 14:6978

Marsh D (1972) Sex pheromone in the aphid Megoura viciae. Nature 238:31-32

Marsh D (1973) Studies on the sex pheromones of the vetch aphid, Megoura viciae. Ph.D. Thesis, University of London

Marsh D (1975) Responses of male aphids to the female sex pheromone in Megoura viciae Buckton. J Ent (A) 50:43-64

Pettersson J (1970a) An aphid sex attractant I. Biological studies. Entomol Scand 1:63-73

Pettersson J (1970b) Studies on Rhopalosiphum padi (L.) I. Laboratory studies on olfaction responses to the winter host Prunus padus L. Lantbrukshogsk Annlr 36:381-399

Pettersson J (1971) An aphid sex attractant II. Histological, ethological and comparative studies. Entomol Scand 2:81-93

Sakuma M, Fukami H (1985) The linear track olfactometer: an assay device for taxes of the German cockroach, Blattella germanica (L.) (Dictyoptera: Blattellidae) toward their aggregation pheromone. Appl Entomol Zool 20:387-402

Steffan AW (1990) Courtship behaviour and possible pheromone spread by hindleg raising in sexual females of aphids (Homoptera: Aphidinea). Entomol Gener 15:33-49

Tamaki G, Butt BA, Landis BJ (1970) Arrest and aggregation of male Myzus persicae (Hemiptera: Aphididae). Ann Entomol Soc Am 63:955960

Tamaki Y (1985) Sex pheromones. Pp 145-191 in Kerkut GA \& Gilbert LI (eds) Comprehensive Insect Physiology Biochemistry and Pharmacology. Vol. 9. Oxford: Pergamon Press 